\title{
Two Stochastic Multidimensional Choice Models for Marketing Research
}

J. Douglas Carroll

Room 2C-247, AT\&T Bell Laboratories, 600 Mountain Avenue, Murray Hill, NJ 07974

Geert De Soete

Department of Psychology, University of Ghent, Henri-Dunantlaan 2, Ghent, Belgium, B-9000

Wayne S. DeSarbo

Marketing and Statistics Departments, Graduate School of Business, University of Michigan, Ann Arbor, MI 48109

\begin{abstract}
Two recently developed probabilistic multidimensional models for analyzing pairwise choice data are introduced, discussed in terms of their differential properties, and extended in several ways. The first one, the wandering vector model, was originally suggested by Carroll [12] and extended by De Soete and Carroll [30]. The second model, called the wandering ideal point model, is a more recently proposed [32] unfolding analog of the wandering vector model. A general maximum likelihood estimation method for fitting the various models described is mentioned, as well as a statistical test for assessing the goodness of fit. Finally, an application of the models is provided concerning consumer choice for some 14 brands of over-the-counter analgesics to illustrate how such models can be gainfully utilized for marketing decision making concerning product positioning.
\end{abstract}

Subject Areas: Consumer Behavior, Marketing Research, and Product Design and Performance.

\section{INTRODUCTION}

The method of paired comparisons typically involves the presentation of stimulus pairs to one or more subjects. The basic experimental unit is the comparison of two stimuli (e.g., products or brands), A and B, by a single subject (e.g., consumer) who, in the simplest case, must choose between them [22]. This method was introduced by Fechner [34] and considerable extensions were made popular by Thurstone [55]. Since this paper is concerned with understanding consumer choice behavior in a marketing context, we will use the terminology of consumers for subjects and products/brands for stimuli. The method of paired comparisons is gainfully utilized in situations where the products to be compared can only be judged subjectively, that is, when it is impossible to make relevant continuous measurement in order to decide which of two products is preferable and by how much. In many cases it is quite feasible for human subjects to make reasonably reliable judgments about the direction, if not the degree, of preference between two such stimuli (although these judgments may be affected by both interand intraindividual differences of the types assumed in the models described in this paper). The most frequent applications in marketing have been taste testing, consumer discrimination tests, quality comparisons, preference, and choice behavior [22]. For $N$ products and $M$ consumers, the total number of paired comparisons will be $M\left({ }_{2}^{N}\right)$, although a number of incomplete designs are also available [9] [10] for reducing the total number of pairwise judgments under simplifying sets of assumptions. Note that when $N$ is large, the task of making consistent pairwise judgments becomes difficult. Often, intransitivities or circular triads occur in such data where, for example, $A$ may be preferred to $B$, and $B$ preferred to $C$, but the same consumer claims to prefer $C$ to $A$. Such inconsistencies suggest that probabilistic or stochastic models may be needed to analyze these paired comparisons data. 
This paper presents two recently developed stochastic multidimensional scaling (MDS) choice models to accommodate the spatial analysis of paired comparisons data for problems in marketing research. We first introduce some considerations that motivate the development of such models. Next, the two stochastic MDS choice models are described in considerable detail. Several special cases that are potentially of interest to marketing researchers are derived, including a model with common and unique dimensions that is shown to be equivalent to a model proposed earlier in [32]. The invariance properties of these models as well as the degrees of freedom are fully discussed for the first time. An illustration of the models is described concerning student paired-comparison choice judgments for some 14 existing major over-the-counter brands of analgesics. The paper concludes with a discussion of potential marketing applications and directions for future research.

\section{SOME EMPIRICAL AND PRACTICAL CONSIDERATIONS}

\section{Inconsistent Choice Behavior}

It is well known that if we repeatedly present the same set of brands to a consumer in an identical situation he or she will not always respond in a consistent manner. As Tversky [58] remarked, a subject often experiences considerable uncertainty when making preference judgments. One way to model this inconsistency is to conceive each response as an event in a probability space leading to the development of stochastic choice models. These models are intended to predict the probabilities of selecting each brand from an offered set (called a feasible set) as the most preferred alternative. We will focus on evaluations of feasible sets with two alternatives per set.

Although in principle it is possible to construct models that account for choices on feasible sets consisting of more than two alternatives, we restrict ourselves to probabilistic choice models for pairwise choices for two reasons. It is far from easy to calculate the choice probabilities predicted by a model for feasible sets larger than two brands because it involves numerical evaluations of multiple integrals. Furthermore, enormous amounts of data are required to fit such models. This makes fitting these models highly impractical in many marketing research situations; therefore, we resort to the time-honored method of paired comparisons as a data gathering procedure. Models fit to such paired comparisons data can then be used to predict choice probabilities from larger feasible sets (e.g., predicting probabilities of first choices in an actual purchase situation in which all alternatives are presented as options).

\section{Similarity Effects}

The two most popular psychological models for representing paired comparisons data are Thurstone's [55] law of comparative judgment (LCJ) case $\mathrm{V}$ and the Bradley-Terry-Luce (BTL) [11] [38] models. To denote the probability that brand $a$ is preferred to brand $b$ by $p(a, b)$, Thurstone's LCJ case V model can be written as

$$
p(a, b)=\phi[u(a)-u(b)]
$$

where $\phi$ is the standard normal distribution function and $u(a)$ is the utility of brand $a$. The BTL model is usually presented as 


$$
p(a, b)=\frac{v(a)}{v(a)+v(b)}
$$

where $v$ is a positive real-valued function on $\{a, b, \ldots\}$. If we define $u(a)=$ $\log [v(a)]$, (2) becomes

$$
p(a, b)=\psi[u(a)-u(b)]
$$

where $\psi$ denotes the standard logistic distribution function. We see from (1) and (3) that Thurstone's LCJ case V and the BTL models are very similar; in fact, the standard normal and logistic distribution functions are so similar in form that enormous numbers of trials are required to distinguish between the two models statistically. Both models imply the following condition commonly referred to as strong stochastic transitivity:

$$
\begin{aligned}
& \text { if } p(a, b) \geq 1 / 2 \text { and } p(b, c) \geq 1 / 2, \\
& \text { then } p(a, c) \geq \max \{p(a, b), p(b, c)\} \text {. }
\end{aligned}
$$

Unfortunately, there is ample experimental evidence [5] [37] [44] [48] [49] [50] [51] [59] [60] that empirical choice proportions often violate strong stochastic transitivity in a systematic way. Empirical choice proportions seem to be influenced not only by differences in utility between the choice objects (e.g., brands), but also to some extent by the similarity or comparability of the choice alternatives. Consumers tend to be somewhat indifferent about very dissimilar alternatives, even when the brands differ considerably in utility. However, similar brands tend to evoke more extreme choice proportions, even when they do not differ very much in utility.

Although empirical choice proportions were often found to violate (4), they usually satisfied a less restrictive transitivity condition known as moderate stochastic transitivity. Moderate stochastic transitivity states that

$$
\begin{aligned}
& \text { if } p(a, b) \geq 1 / 2 \text { and } p(b, c) \geq 1 / 2 \text {, } \\
& \text { then } p(a, c) \geq \min \{p(a, b), p(b, c)\} \text {. }
\end{aligned}
$$

It can be proved that any model of the form

$$
p(a, b)=F\left(\frac{u(a)-u(b)}{d^{*}(a, b)}\right)
$$

where $F$ is monotonically increasing with $F(x)=1-F(-x), u$ is a real-valued function on $\{a, b, \ldots\}$, and $d^{*}$ is a (semi-)metric implies (5) but not necessarily (4) [35]. A model of the form (6) is called a moderate utility model. Contrary to models that imply (4), moderate utility models can account for the empirically observed similarity effects. Consequently, to model the empirically collected paired comparison judgments more accurately, a pairwise choice model should be a moderate utility model. In this paper, we concentrate on probabilistic, multidimensional choice models to represent paired comparisons data that are moderate utility models. 


\section{Unavailable A Priori Psychological Information}

Because of the similarity effects mentioned above, various moderate utility models have been proposed in the psychological literature in recent years [12] [33] [36] [39] [53] [54] [59] [60]. Several of these models were inspired by Restle's [43] set-theoretic choice model and require a priori information about the psychological feature structure of the choice objects (e.g., brands) in order to be applicable. However, in many applied settings this a priori information is not available and these models cannot be utilized. In addition, in many product classes with strong brand name effects, features/attributes may not adequately describe choice behavior. In order to be routinely applicable in a variety of marketing research situations, a choice model should not necessarily require a priori psychological information about the choice objects.

\section{LITERATURE REVIEW}

The psychometric literature provides a number of spatial models to display the structure in such paired comparisons data when representing consumers and brands. There have been a number of unidimensional scaling procedures proposed to obtain scale values for brands from such (aggregated) paired comparisons data [9] [55] [56]. More recently, MDS have been devised to account for the multidimensional nature of the brands. Two general classes of models have been typically utilized to represent such preference/choice data: vector and unfolding models. A vector or scalar products MDS [52] [57] represents consumers as vectors and brands as points in an $r$-dimensional space. The projection of brand points onto subject vector(s) is assumed to be monotone with that subject's preference. The goal of the analysis is to estimate the optimal vector directions and brand coordinates in a prescribed dimensionality. An intuitively unattractive property of the vector model is that it assumes preference or utility to change monotonically with all dimensions; that is, it assumes that if a certain amount of a thing is good, even more must be even better. As argued by Coombs [18] and Carroll [12], this is not an accurate representation for most quantities or attributes in the real world (perhaps with the exceptions of health, wealth, wisdom, and happiness).

There has been some work on analyzing paired comparisons via such vector or scalar products models. Bechtel, Tucker, and Chang [4] developed a scalar products model in which they imposed restrictions on sums and variances and constraints on various parameters to insure uniqueness of the solution (e.g., orthogonality). Cooper and Nakanishi [20] developed two logit models (vector and ideal point) for the external analysis of paired comparisons data (see also [26]). By external analysis we mean an analysis in which the stimulus or brand coordinates are given a priori so that only the parameters for subjects or consumers must be estimated. The models and methods focused on in this paper imply internal analysis, in which both subject and stimulus parameters are estimated from the data.

The other major type of psychometric model to represent such preference/ choice data is the unfolding model [18]. We will discuss only the simple unfolding model of Coombs [18] (see [12] and [13] for a discussion of the simple, weighted, and general unfolding models, and the work by DeSarbo and Rao [27] [28] on GENFOLD2, an unfolding methodology that accommodates the estimation of all three types of unfolding models). In the simple unfolding model, both consumers and brands are represented as points in an r-dimensional space. The points for consumers represent ideal products or optimal sets of dimension values. As 
formulated originally by Coombs in the unidimensional case [17] and as generalized to the multidimensional case by Bennett and Hays [6], the farther a given brand point is from a consumer's ideal point, the less utility that consumer has for that brand. This notion of relative distance implies an often but not necessarily Euclidean metric on the space. Following the formulation of multidimensional unfolding by Bennett and Hays [6], we explicitly assume this metric to be Euclidean. This implies that iso-utility contours are families of concentric circles centered at a consumer's ideal point in $r=2$ dimensions. Carroll [12] demonstrated that the vector model is a special case of this unfolding model where the ideal points go off to infinity. In unfolding analyses, the consumer's preference is a function of the distance away from the respective ideal point. The objective of unfolding analyses is to estimate the optimal set of ideal points and brand coordinates in a prescribed dimensionality.

Although several unidimensional stochastic unfolding models have been proposed in the literature [2] [3] [19] [47] [63], few multidimensional probabilistic unfolding models have been developed to accommodate paired comparisons data. The first approach by Schönemann and Wang [45] and Wang, Schönemann, and Rusk [61] is based on the well-known BTL model and consequently assumes strong stochastic transitivity. In the multidimensional unfolding model proposed by Zinnes and Griggs [63], it is assumed that the coordinates of both the consumer and the brand points are independently normally distributed with a common variance. Zinnes and Griggs assume that for each element of the brand pair a consumer independently samples a point from his or her ideal point distribution (see also [25]). As demonstrated in [32], the Zinnes and Griggs model is a strong utility model.

Another large class of choice models that is widely used in econometrics (and in marketing) includes the multinomial logit (MNL) [40] and the multinomial probit (MNP) [21] [41] [42] models. While the MNL models are strong utility models, many of the MNP models entail moderate (but not necessarily strong) stochastic transitivity and so are generally moderate utility models. However, the estimation procedures developed by econometricians for fitting these models to various types of choice data generally assume that the stimulus dimensions (or attributes of the alternatives) are given a priori. No procedures have been devised for estimating simultaneously all the relevant parameters of most models in this class. One special case of the MNP model, the random coefficients multinomial probit (RCMNP) model, is mathematically equivalent to the wandering vector model discussed in the next section, although the RCMNP model assumes random variation of coefficients over subjects in a sample from some population (i.e., interindividual variation) while the wandering vector model assumes intraindividual variation. Thus, the procedure described by Carroll and De Soete [30] and discussed in a broader context here can be viewed as the first method for estimating all necessary parameters for full specification of this model from (paired comparison) choice data alone (i.e., without requiring a priori information about either the attributes of the alternatives or demographic or other information on the subjects making these judgments). It should be noted that paired comparisons data collected at the individual level is not a common type of data in economics and is sometimes hard to gather in practical marketing studies. However, under the assumptions usually made in economics (and in many marketing studies) the models can be fit to paired comparisons data amalgamated over a group or subgroups of subjects. (This will be discussed in more detail later.) See [42] for 
a comprehensive review of these and other economic choice models and their relation to many of the psychometric choice models discussed above, as well as potential applications of these models to market research.

In the next two sections, two recently developed multidimensional choice models are described. These models take into account the three considerations discussed above; they are probabilistic, imply only moderate stochastic transitivity, and do not require any a priori psychological information about the choice objects. In addition, they can in principle be applied to three-way paired comparisons data (i.e., different paired comparisons matrices about the same brands).

\section{THE WANDERING VECTOR MODEL}

The wandering vector (WV) model introduced by Carroll [12] and generalized by De Soete and Carroll [30] is a probabilistic version of the well-known vector model [52] [57] for analyzing choice data. An even more general formulation of the $\mathrm{WV}$ model is given in [31] and is presented in the next section.

\section{General Formulation}

The WV model seeks a joint representation of consumers (or groups of consumers) and brands in an $r$-dimensional space. Each consumer $i(i=1, N)$ is represented by a vector emanating from the origin with a terminus $\mathbf{Y}_{i}$ that follows a multivariate normal distribution (mean $=\mu_{i}$, covariance matrix $=\Sigma_{i}$ ):

$$
\mathbf{Y}_{i} \sim N\left(\mu_{i}, \Sigma_{i}\right)
$$

It is assumed that distributions of the $N$ consumer vectors are independent of each other; that is,

$$
\operatorname{Covar}\left(\mathbf{Y}_{i}, \mathbf{Y}_{i^{\prime}}\right)=0
$$

for $i, i^{\prime}=1, N$, and $i \neq i^{\prime}$. Each brand $j(j=1, M)$ is represented as a fixed point $\mathbf{x}_{j}$ in the same multidimensional space.

According to the model, a consumer $i$ samples $\mathbf{y}_{i}$ from $\mathbf{Y}_{i}$ each time he or she is presented a pair of brands $(j, k)$. Alternative $j$ is preferred to $k$ whenever the orthogonal projection of $x_{j}$ on the vector from the origin to $\mathbf{y}_{i}$ exceeds the orthogonal projection of $x_{k}$ on that vector, that is, whenever

$$
\frac{\mathbf{x}_{j}{ }^{\prime} \mathbf{y}_{i}}{\| \mathbf{y}_{i}||}>\frac{\mathbf{x}_{k}{ }^{\prime} \mathbf{y}_{i}}{|| \mathbf{y}_{i} \|} \text {. }
$$

Since a new $\mathbf{y}_{i}$ is sampled each time the consumer is presented a pair of brands, a consumer need not always respond consistently on repeated presentations of the same brand pair.

Multiplication of both sides of (8) by $\| \mathbf{y}_{i}||$ shows that consumer $i$ prefers brand $j$ to $k$ whenever

$$
\mathbf{x}_{j}{ }^{\prime} \mathbf{y}_{i}>\mathbf{x}_{k}{ }^{\prime} \mathbf{y}_{i}
$$

Consequently, the probability that consumer $i$ prefers brand $j$ to $k$ (written as $p_{i j k}$ ) is 


$$
\begin{aligned}
p_{i j k} & =\operatorname{Prob}\left\{\mathbf{x}_{j}{ }^{\prime} \mathbf{Y}_{i}>\mathbf{x}_{k} \mathbf{Y}_{i}\right\} \\
& =\operatorname{Prob}\left\{\left(\mathbf{x}_{j}-\mathbf{x}_{k}\right)^{\prime} \mathbf{Y}_{i}>0\right\} .
\end{aligned}
$$

Since it follows from (7) that

$$
\left.\left(\mathbf{x}_{j}-\mathbf{x}_{k}\right)^{\prime} \mathbf{Y}_{i} \sim N\left(\mathbf{x}_{j}-\mathbf{x}_{k}\right)^{\prime} \mu_{i}, \delta_{i j k}^{2}\right)
$$

with

$$
\delta_{i j k}^{2}=\left(\mathbf{x}_{j}-\mathbf{x}_{k}\right)^{\prime} \Sigma_{i}\left(\mathbf{x}_{j}-\mathbf{x}_{k}\right)
$$

(9) becomes

$$
p_{i j k}=\phi\left(\frac{\left(\mathbf{x}_{j}-\mathbf{x}_{k}\right)^{\prime} \mu_{i}}{\delta_{i j k}}\right),
$$

which constitutes the general formulation of the WV model. When $N=1$, the WV model can be shown to be a special case of a Thurstonian choice model proposed by Heiser and de Leeuw [36] and Takane [54] (also see [29]).

\section{Properties}

To show that the WV model is a moderate utility model, we can rewrite (10) as

$$
p_{i j k}=\phi\left(\frac{u_{i j}-u_{i k}}{\delta_{i j k}}\right)
$$

with $u_{i j}=\mathbf{x}_{j}{ }^{\prime} \mu_{i}$.

Since a covariance matrix $\Sigma_{i}$ is always positive (semi-)definite, $\delta_{i j k}$ is a (semi-)metric (i.e., the $\delta_{i j k}$ are nonnegative, symmetric, and satisfy the triangle inequality) and (11) is of the form (6).

The following counterexample illustrates that the choice probabilities generated by the WV model do not necessarily satisfy strong stochastic transitivity. Let $\mu_{i}=(1,1 / 3)^{\prime}, \Sigma_{i}=I$ (the identity matrix), $\mathbf{x}_{j}=(1 / 3,2)^{\prime}, \mathbf{x}_{k}=(2 / 3,1)^{\prime}$, and $\mathbf{x}_{l}=$ $(1 / 3,2 / 3)$; then $p_{i j k}=.50$ and $p_{i k l}=.83$, but $p_{i j l}=.63$.

\section{Degrees of Freedom of the WV Model}

In its general form, the WV model has $M_{r}+N[r+r(r+1) / 2]$ parameters. However, the model does not determine all these parameters uniquely. More specifically, the choice probabilities defined by $(10)$ remain invariant under the following transformations of the parameters.

1. Dilation of the consumer vectors

If we replace

$$
\mathbf{Y}_{i} \sim N\left(\mu_{i}, \Sigma_{i}\right)
$$

by

$$
\alpha_{i} \mathbf{Y}_{i} \sim N\left(\alpha_{i} \mu_{i}, \alpha_{i}^{2} \Sigma_{i}\right)
$$


where $\alpha_{i}$ is an arbitrary positive constant, the choice probabilities are not affected.

2. Translation of the brand points

If the transformation

$$
\mathbf{x}_{j} \rightarrow \beta+\mathbf{x}_{j}
$$

where $\beta$ is an arbitrary $r$-component vector, is applied to every brand point $\mathbf{x}_{j}(j=1, M)$, the choice probabilities are left invariant.

3. Nonsingular transformation of the brand points and consumer vectors If $\mathbf{Q}$ is an arbitrary $r \times r$ matrix such that $\mathbf{Q}^{-1}$ exists, then the choice probabilities are not affected by the following transformations:

$$
\begin{aligned}
& \mathbf{x}_{j} \rightarrow \mathbf{Q}^{\prime} \mathbf{x}_{j}, \\
& \mathbf{Y}_{i} \rightarrow \mathbf{Q}^{-1} \mathbf{Y}_{i},
\end{aligned}
$$

where

$$
\mathbf{Q}^{-1} \mathbf{Y}_{i} \sim N\left(\mathbf{Q}^{-1} \mu_{i}, \mathbf{Q}^{-1} \Sigma_{i}\left(\mathbf{Q}^{-1}\right)^{\prime}\right)
$$

Because of these indeterminacies, the degrees of freedom of the general WV model reduce to

$$
M_{r}+N[r+r(r+1) / 2]-N-r-r^{2}=M_{r}+N[r(r+3) / 2-1]-r-r^{2} .
$$

\section{Special Cases}

In empirical applications, it might be interesting to impose restrictions on the general model to verify specific hypotheses. The validity of a hypothesis can then be tested by statistically comparing the fit of the restricted model with the fit of the general model.

De Soete and Carroll [30] considered a special case in which the variancecovariance matrices of the subject vector termini are restricted to be proportional to each other:

$$
\Sigma_{i}=c_{i} \Sigma\left(c_{i}>0\right)
$$

Because of the indeterminacy expressed in (12), $\Sigma$ can be set equal to an identity matrix without loss of generality. Defining $\mu_{i}^{*}$ as $\mu_{i} / \sqrt{ } c_{i}$, this restricted model can be written as

$$
p_{i j k}=\phi\left(\frac{\left(\mathbf{x}_{j}-\mathbf{x}_{k}\right)^{\prime} \mu_{i}^{*}}{d\left(\mathbf{x}_{j}, \mathbf{x}_{k}\right)}\right),
$$

where $d(.,$.$) denotes the Euclidean distance function$

$$
d^{2}\left(\mathbf{x}_{j}, \mathbf{x}_{k}\right)=\left(\mathbf{x}_{j}-\mathbf{x}_{k}\right)^{\prime}\left(\mathbf{x}_{j}-\mathbf{x}_{k}\right)
$$

The degrees of freedom of this restricted model are

$$
(M+N) r-r(r+1) / 2-1 .
$$


When $N=1$, (16) equals (13) showing that (14) only imposes real constraints on the general model when $N>1$. The $\Sigma_{i}$ can also be constrained to be diagonal; because of the indeterminacy stated in (12), this case only imposes real constraints on the general model when $N>2$. The degrees of freedom for this model are

$$
M r+N(2 r-1)-2 r
$$

Note that (13) exceeds (17) only when $N>2$.

In the general model, De Soete and Carroll [30] introduced an additional error term to account for response variability due to unaccounted-for variance associated with dimensions not present in the model. This extended model can be shown to be a special case of the general model. Suppose, in analogy to the factor analysis model, that the $M$ brands have $r(<M)$ dimensions in common and that there is a specific dimension for each brand. Therefore, the brand coordinates can be written as

$$
\mathbf{X}^{*}=\left(\mathbf{X} \mathbf{I}_{M}\right),
$$

where $\mathbf{X}=\left(\mathbf{x}_{1}, \ldots, \mathbf{x}_{M}\right)^{\prime}$ contains the coordinates of the $M$ brands on $r$ common dimensions and $\mathbf{I}_{M}$ is an identity matrix of order $M$. Assume that $\mathbf{Y}_{i}{ }^{*}$, the $r+M$ dimensional random variable representing the terminus of the $i$ th consumer vector, is distributed as follows:

$$
\mathbf{Y}_{i}^{*} \sim N\left(\left[\begin{array}{l}
\mu_{i} \\
M^{\mathbf{0}_{1}}
\end{array}\right],\left[\begin{array}{ll}
\Sigma_{i} & r_{M} \\
M^{\mathbf{0}_{r}} & \gamma_{i}{ }^{2} \mathbf{I}_{M}
\end{array}\right]\right)
$$

where $s^{0}$ denotes an $s \times t$ matrix filled with zeros (i.e., $\mathbf{Y}_{i}{ }^{*}$ is assumed to have variance $\gamma_{i}^{2}$ along each specific dimension. Since

$$
\left(\mathbf{x}_{j}^{*}-\mathbf{x}_{k}^{*}\right)^{\prime} \mathbf{Y}_{i}^{*}-N\left(\left(\mathbf{x}_{j}-\mathbf{x}_{k}\right)^{\prime} \mu_{i}, \delta_{i j k}^{2}+2 \gamma_{i}^{2}\right),
$$

the model becomes

$$
p_{i j k}=\phi\left(\frac{\left(\mathbf{x}_{j}-\mathbf{x}_{k}\right)^{\prime} \mu_{i}}{\sqrt{ } \delta_{i j k}^{2}+2 \gamma_{i}^{2}}\right)
$$

This model is equivalent to a model proposed earlier by De Soete and Carroll [30]. Note that (19) can be generalized by allowing for nonzero expectations on the specific dimensions as well as for differential variances along the specific dimensions. The latter extension is closely related to a general model for proximity data proposed by Winsberg and Carroll [62] called the extended two-way Euclidean model, which was generalized to the three-way (or individual differences) case (called the extended INDSCAL model) by Carroll and Winsberg [15].

\section{THE WANDERING IDEAL POINT MODEL}

The geometric representation on which the WV model is based assumes that all consumers have a monotone preference function on each dimension; that is, it is assumed that the larger the value of a brand on a dimension, the more the brand 
will be preferred. This is true for some brand attributes but certainly not for all attributes. For example, if the brands are coffee brands varying in bitterness, a consumer probably has a most preferred bitterness level. The farther away the bitterness of a coffee brand is from this optimum level, the less it will be preferred. This implies that the consumer does not have a monotone but a singlepeaked preference function on the bitterness dimension.

A model that allows for single-peaked preference functions is Coombs' [18] unfolding model. In the next section, we present a probabilistic unfolding model, called the wandering ideal point (WIP) model, that was recently proposed by De Soete, Carroll, and DeSarbo [32].

\section{General Formulation}

In the WIP model, both the consumers and brands are presented as points in a joint $r$-dimensional Euclidean space. Whereas the brands $1,2, \ldots, M$ are represented by fixed points $x_{1}, x_{2}, \ldots, x_{M}$, the consumers are represented by random points. More specifically, each consumer $i(i=1, N)$ is represented by a random point $\mathbf{Y}_{i}$, which follows a multivariate normal distribution

$$
\mathbf{Y}_{i} \sim N\left(\mu_{i}, \Sigma_{i}\right)
$$

As with the WV model, it is assumed that the distributions of the $N$ consumer points are independent of each other; that is,

$$
\operatorname{Covar}\left(\mathbf{Y}_{i}, \mathbf{Y}_{i^{\prime}}\right)=\mathbf{0}
$$

for $i, i^{\prime}=1, N$ and $i \neq i^{\prime}$.

Each time a pair of brands $(j, k)$ is presented to a consumer $i$, he/she samples a point $\mathbf{y}_{i}$ from $\mathbf{Y}_{i}$. In accordance with Coombs' [18] unfolding theory, the consumer prefers $j$ to $k$ whenever

$$
d\left(\mathbf{y}_{i}, \mathbf{x}_{j}\right)<d\left(\mathbf{y}_{i}, \mathbf{x}_{k}\right)
$$

with $d(.,$.$) defined in (15). Because a consumer always chooses the brand that is$ closest to $\mathbf{y}_{i}, \mathbf{y}_{i}$ can be considered consumer $i$ 's ideal point. However, since a new $\mathbf{y}_{i}$ is sampled each time a pair of brands is presented, the consumer's ideal point is not fixed but "wanders" from trial to trial-hence the model's name.

By squaring both sides of (22) and rearranging terms, we determine that consumer $i$ prefers $j$ to $k$ whenever

$$
\left(\mathbf{x}_{k}-\mathbf{x}_{j}\right)^{\prime} \mathbf{y}_{i}<\left(\mathbf{x}_{k}{ }^{\prime} \mathbf{x}_{k}-\mathbf{x}_{j}{ }^{\prime} \mathbf{x}_{j}\right) / 2
$$

Therefore, the probability that consumer $i$ prefers object $j$ to $k$ is

$$
p_{i j k}=\operatorname{Prob}\left\{\left(\mathbf{x}_{k}-\mathbf{x}_{j}\right)^{\prime} \mathbf{Y}_{i}<\left(\mathbf{x}_{k}{ }^{\prime} \mathbf{x}_{k}-\mathbf{x}_{j}{ }^{\prime} \mathbf{x}_{j}\right) / 2\right\} \text {. }
$$

It follows from (21) that

$$
\left(\mathbf{x}_{k}-\mathbf{x}_{j}\right)^{\prime} \mathbf{Y}_{i} \sim N\left(\left(\mathbf{x}_{k}-\mathbf{x}_{j}\right)^{\prime} \mu_{i}, \delta_{i j k}^{2}\right)
$$

Consequently, (23) becomes 


$$
p_{i j k}=\phi\left(\frac{\left(\mathbf{x}_{k}{ }^{\prime} \mathbf{x}_{k}-\mathbf{x}_{j}{ }^{\prime} \mathbf{x}_{j}\right)-2\left(\mathbf{x}_{k}-\mathbf{x}_{j}\right)^{\prime} \mu_{i}}{2 \delta_{i j k}}\right) .
$$

Equation (24) provides the general formulation of the WIP model.

\section{Properties}

Again, it is straightforward to prove that the WIP model is a moderate utility model. By defining

$$
u_{i j}=\mathbf{x}_{j}{ }^{\prime} \mu_{i}-\mathbf{x}_{j}{ }^{\prime} \mathbf{x}_{j} / 2
$$

(24) can be written as

$$
p_{i j k}=\phi\left(\left(u_{i j}-u_{i k}\right) / \delta_{i j k}\right) .
$$

By using a simple counterexample we show that the choice probabilities defined by the WIP model do not necessarily satisfy strong stochastic transitivity. Let $\mu_{i}=(0,0)^{\prime}, \Sigma_{i}=\mathbf{I}, \mathbf{x}_{j}=(3,1)^{\prime}, \mathbf{x}_{k}=(4,0)^{\prime}$, and $\mathbf{x}_{l}=(-5,0)^{\prime} ;$ then $p_{i j k}=.98$ and $p_{i k l}=.69$, but $p_{i j l}=.82$.

\section{Degrees of freedom of the WIP model}

Just like the WV model, in its general form the WIP model has $M r+N r+$ $r(r+1) / 2$ ] parameters. Again, all these parameters are not uniquely determined by the model. More precisely, the choice probabilities defined by (25) are invariant under the following parameter transformations:

1. Translation of the consumer and brand points

Adding the same arbitrary $r$-component vector to all consumer and brand points does not affect the choice probabilities.

2. Central dilation of the consumer and the brand points The transformations

$$
\begin{array}{ll}
\mathbf{x}_{j}-\alpha \mathbf{x}_{j} & (i=1, M) \\
\mathbf{Y}_{i} \rightarrow \alpha \mathbf{Y}_{i} & (i=1, N),
\end{array}
$$

where $\alpha$ is an arbitrary positive constant, leaves the choice probabilities invariant. Note that

$$
\alpha \mathbf{Y}_{i} \sim N\left(\alpha \mu_{i}, \alpha^{2} \Sigma_{i}\right) .
$$

3. Orthogonal rotation of the consumer and brand points

Application of the same orthogonal rotation $\mathbf{T}$ to all consumer and brand points does not affect the choice probabilities predicted by the model. Note that the distribution of $\mathbf{T Y} \mathbf{Y}_{i}$ is

$$
\mathbf{T} \mathbf{Y}_{i} \sim N\left(\mathbf{T} \mu_{i}, \mathbf{T} \Sigma_{i} \mathbf{T}^{\prime}\right) .
$$

By subtracting the number of parameters needed to specify these indeterminacies from the total number of parameters, we obtain the degrees of freedom of the general WIP model: 


$$
(M+N) r+N r(r+1) / 2-r(r+1) / 2-1 .
$$

\section{Special Cases}

As with the WV model, it might be interesting to impose certain restrictions on the general model. First, various kinds of restrictions can be imposed on the variance-covariance matrices of the consumer points. The $\Sigma_{i}$ can, for instance, be constrained to be diagonal. Due to the rotational indeterminacy of the general model, setting the off-diagonal elements of the variance-covariance matrices equal to zero only imposes real constraints on the general model when $N>1$. The degrees of freedom for this model are

$$
(M+2 N) r-r-1
$$

A more restrictive case constrains all $\Sigma_{i}$ to be identity matrices. In this case, the model has $(M+N) r-r(r+1) / 2$ degrees of freedom.

Analogously to the last special case discussed in the context of the WV model, we could assume a specific dimension for each brand besides $r(<M)$ common dimensions. If the brand coordinates can be written as in (18) and if the consumer ideal points are distributed as in (19), the WIP model becomes

$$
p_{i j k}=\phi\left(\frac{\left(\mathbf{x}_{k}{ }^{\prime} \mathbf{x}_{k}-\mathbf{x}_{j}{ }^{\prime} \mathbf{x}_{j}\right)-2\left(\mathbf{x}_{k}-\mathbf{x}_{j}\right)^{\prime} \mu_{i}}{2 \sqrt{ } \delta_{i j k}^{2}+2 \gamma_{i}^{2}}\right) \text {. }
$$

DeSarbo, De Soete, and Eliashberg [25] proposed a random utility alternative to the WIP model allowing for reparameterization options on $\mu_{i}$ and/or $\mathbf{x}_{j}$ as functions of specified background variables/attributes.

In order to apply the WV and WIP models described above, replicated paired comparisons data must be collected from one or more consumers or groups of consumers. On the basis of these data, the model parameters need to be estimated. Here, maximum likelihood estimation using Fisher's scoring method has been quite satisfactory for estimating the parameters of certain special cases of the WV and WIP models [30] [32]. Maximum likelihood estimation has the advantage of enabling model selection by means of likelihood ratio tests. However, likelihood ratio statistics can only be constructed for comparing the fit of nested models. When nonnested models are to be compared, an information criterion can be used such as those proposed by Akaike [1], Chow [16], and Schwarz [46].

\section{ILLUSTRATION}

\section{Study Description}

Thirty undergraduate students in a marketing research class at the University of Pennsylvania were paid to take part in a smail study designed to measure preferences for various brands of existing over-the-counter (OTC) analgesic pain relievers. These respondents were requested to complete a questionnaire that measured the brand(s) currently used (as well as frequency of use), the importance of self-explicated attributes, usage occasions, and several psychographic questions.'

\footnotetext{
'The questionnaire had been pretested on a similar student sample of ten students for wording, content, and length.
} 
In addition, the questionnaire presented 14 existing OTC analgesic brands listed in Table 1. Initially, the students were presented color photographs of each brand and its packaging, price per 100 tablets, ingredients, package claims, and manufacturer [23] [27]. ${ }^{2}$ Table 1 presents summaries of selected portions of the descriptions for each of the 14 brands. In this part of the questionnaire, each student/consumer subject was requested to read the information contained on these color photographs and return to them at any time during the experiment if he/she so desired. After a period of time, each subject was asked to make paired comparison preference judgments for all 91 pairs of brands. The subjects were told to choose one from each pair (i.e., no ties were allowed). The presented pairs were randomized for each questionnaire/subject. The survey was completed outside of class so there was no explicit time pressure on the students. Given the small convenience sample of students and the "unnatural" experimental setting, we do not make any inferences about the nature of the analgesics' market at large but wish solely to provide an illustration of the methodology.

\section{Analysis}

These data were analyzed according to WV and WIP models using maximum likelihood estimation, the results of which are summarized in Table 2 . The null model refers to a model in which a binomial probability is estimated for each pair of brands with no structural constraints on these parameters. This fully saturated model is the most general model possible for paired comparisons data. Four competing WV and WIP models were considered and are described in Table 2. Of these four models, the WV model with unique dimensions as defined in (20) possesses the minimum Akaike information criterion (AIC) statistic [1] and is therefore the solution we choose to discuss. Note that the two-dimensional WV model with unique dimensions has the same degrees of freedom as the WIP model with an identity variance-covariance matrix.

Figure 1 presents this $T=2$ dimensional WV solution, in which the brands are labeled by the letters $A$ through $\mathbf{N}$ and the preference vector is designated by the solid line with an asterisk at its terminus. To aid in subsequent interpretation, we also related (via multiple regression) the attributes listed in Table 1 to the brand coordinates (see other approaches in [7], [8], [14], [25], and [26]). These vectors, labeled $P$ through $V$, are normalized to constant length for convenience. Note that the attributes in Table 1 account for 81.2 percent of the variation of dimension one and 85.6 percent of the variation of dimension two. A canonical correlation analysis of the brand coordinates with these attributes yields canonical correlations of .925 and .895 . These analyses appear to confirm the relatively strong relationship between the product attributes in Table 1 and the derived brand coordinates, indicating the large role of this information in choice judgments. Table 3 presents the correlations of those seven attributes with each of the two derived dimensions (brand coordinates).

The first dimension appears to separate brands containing aspirin from those containing ibuprofen; those brands whose major analgesic ingredient is aspirin tend to load negatively and the two ibuprofen brands (Advil and Nuprin) load positively. It is interesting to note that the acetaminophen brands load near the origin or in the middle of the aspirin and ibuprofen brands. Also note that the dimension

\footnotetext{
${ }^{2}$ The displayed information contained all attributes the pretest sample claimed were important in respondents' choice of an OTC analgesic brand, as well as other attributes cited in [23], [25], and [27].
} 
Table 1: Some OTC analgesic attributes for the 14 brands.

\begin{tabular}{|c|c|c|c|c|c|c|c|}
\hline \multirow[b]{2}{*}{ Brands } & \multicolumn{7}{|c|}{ Attribute Label } \\
\hline & $\begin{array}{c}\mathrm{Mg} \\
\text { Aspirin } \\
\mathrm{P} \\
\end{array}$ & $\begin{array}{c}\mathbf{M g} \\
\text { Acetaminophen } \\
\mathrm{Q}\end{array}$ & $\begin{array}{c}\mathbf{M g} \\
\text { Ibuprofen } \\
\mathbf{R} \\
\end{array}$ & $\begin{array}{c}\mathrm{Mg} \\
\text { Caffeine } \\
\mathrm{S} \\
\end{array}$ & $\begin{array}{c}\mathrm{Mg} \\
\text { Buffered } \\
\text { Ingredients } \\
\mathrm{T}\end{array}$ & $\begin{array}{c}\text { Price } \\
\mathrm{U}\end{array}$ & $\begin{array}{c}\text { Maximum } \\
\text { Dosage } \\
\text { per Day } \\
\mathrm{V} \\
\end{array}$ \\
\hline $\mathrm{A}$ & 0 & 0 & 200 & 0 & 0 & 6.99 & 6 \\
\hline B & 400 & 0 & 0 & 32 & 0 & 3.79 & 10 \\
\hline C & 0 & 500 & 0 & 0 & 0 & 5.29 & 8 \\
\hline D & 325 & 0 & 0 & 0 & 150 & 3.29 & 12 \\
\hline $\mathbf{E}$ & 325 & 0 & 0 & 0 & 0 & 2.69 & 12 \\
\hline $\mathbf{F}$ & 324 & 0 & 0 & 0 & 100 & 3.89 & 10 \\
\hline G & 421 & 0 & 0 & 32 & 75 & 5.31 & 8 \\
\hline $\mathrm{H}$ & 500 & 0 & 0 & 0 & 100 & 1.99 & 12 \\
\hline I & 0 & 500 & 0 & 0 & 0 & 5.75 & 8 \\
\hline J & 250 & 250 & 0 & 65 & 0 & 4.99 & 8 \\
\hline $\mathrm{K}$ & 0 & 0 & 200 & 0 & 0 & 7.59 & 6 \\
\hline $\mathrm{L}$ & 0 & 500 & 0 & 0 & 0 & 4.99 & 8 \\
\hline M & 0 & 325 & 0 & 0 & 0 & 3.69 & 8 \\
\hline $\mathrm{N}$ & 227 & 194 & 0 & 33 & 75 & 4.99 & 12 \\
\hline \multicolumn{8}{|c|}{ Legend for Brand Labels } \\
\hline \multicolumn{2}{|c|}{ Brands } & Label & & & & & \\
\hline \multicolumn{2}{|c|}{$\overline{\text { Advil }}$} & $\overline{\mathbf{A}}$ & & & & & \\
\hline \multicolumn{2}{|c|}{$\begin{array}{l}\text { Advil } \\
\text { Anacin }\end{array}$} & B & & & & & \\
\hline \multicolumn{2}{|c|}{ Anacin-3 } & $\mathrm{C}$ & & & & & \\
\hline \multicolumn{2}{|c|}{ Ascriptin } & D & & & & & \\
\hline \multicolumn{2}{|c|}{ Bayer } & E & & & & & \\
\hline \multicolumn{2}{|c|}{ Bufferin } & $\bar{F}$ & & & & & \\
\hline \multicolumn{2}{|c|}{ Cope } & G & & & & & \\
\hline \multicolumn{2}{|c|}{ CVS (a generic) } & $\mathrm{H}$ & & & & & \\
\hline \multicolumn{2}{|c|}{ Datril } & I & & & & & \\
\hline \multicolumn{2}{|c|}{ Excedrin } & $j$ & & & & & \\
\hline \multicolumn{2}{|c|}{ Nuprin } & K & & & & & \\
\hline \multicolumn{2}{|c|}{ Panadol } & $\mathrm{L}$ & & & & & \\
\hline \multirow{2}{*}{\multicolumn{2}{|c|}{ Tylenol }} & $M$ & & & & & \\
\hline & & $\mathbf{N}$ & & & & & \\
\hline
\end{tabular}

correlates highly with price since the ibuprofen brands generally are much more expensive than their aspirin or acetaminophen counterparts. Similarly, there is a relationship with maximum dosage since the more potent ibuprofen brands require smaller dosage/day recommendations.

The second dimension provides an interesting perspective on the structure of the data. On the basis of the loadings in Table 3, this dimension is related to market share and brand complexity. The less popular and more complex (aspirin) formulations with caffeine and buffered ingredients (to prevent stomach upset often caused by aspirin) such as Cope $(G)$ and Vanquish $(N)$ load on the negative end of this dimension while the higher market share, "simpler" (i.e., no caffeine or buffered ingredients) acetaminophen- and ibuprofen-only brands, tends to load positively (e.g., Tylenol (M), Advil (A), Anacin-3 (C), Panadol (L), and Nuprin (K)).

The preference vector is represented by a solid vector with a terminus labeled by an asterisk $\left(^{*}\right)$ in Figure 1. It indicates that overall preference for this group of subjects tends to be towards brands located near quadrants 1 and 2, including Tylenol, Anacin-3, Bayer, and Panadol. The two dashed vectors in the figure indicate the sector in which this preference vector is expected to fall 68 percent of the time-a confidence region for the estimated vector. Thus, overall preference appears to be towards the simple formulated, more heavily advertised brands of analgesics. 
Table 2: Summary of the analyses on the analgesic data.

\begin{tabular}{|c|c|c|c|c|c|}
\hline \multirow[b]{2}{*}{ Model } & \multirow[b]{2}{*}{ Log-likelihood } & \multirow{2}{*}{$\begin{array}{l}\text { Degrees of } \\
\text { Freedom of } \\
\text { the Model }\end{array}$} & \multirow[b]{2}{*}{ AIC } & \multicolumn{2}{|c|}{$\begin{array}{l}\text { Likelihood Ratio } \\
\text { Test Against } \\
\text { Null Model }\end{array}$} \\
\hline & & & & $x^{2}$ & $d f$ \\
\hline Null model & -1461.8 & 91 & 3105.5 & & \\
\hline $\begin{array}{l}\text { 2-dimensional WV model } \\
\text { as defined in (10) }\end{array}$ & -1484.0 & 26 & 3019.9 & 44.4 & 65 \\
\hline $\begin{array}{l}\text { 2-dimensional WV model } \\
\text { with unique dimensions } \\
\text { as defined in (20) }\end{array}$ & -1475.5 & 27 & 3004.9 & 27.4 & 64 \\
\hline $\begin{array}{l}\text { 2-dimensional WIP model } \\
\text { as defined in (24) }\end{array}$ & -1480.5 & 29 & 3019.0 & 37.4 & 62 \\
\hline $\begin{array}{l}\text { 2-dimensional WIP model } \\
\text { with identity variance- } \\
\text { covariance matrix }\end{array}$ & -1482.0 & 27 & 3018.1 & 40.6 & 64 \\
\hline
\end{tabular}

If this rather homogeneous group of consumers was representative of a target market segment, a manufacturer could use the information provided by this analysis to understand competitive market structure and brand positioning. The locations of the brands in the derived space appear to be ingredients-based. Of particular interest is the manner in which the newer ibuprofen brands (Advil and Nuprin) appear to have created a rather unique niche for themselves in this market (quadrant one) and the appeal they have to this particular market segment given that their projections on the preference vector are higher than those of many aspirin brands. Tylenol is the most preferred brand as predicted from Figure 1, which is interesting given the two tampering episodes that were so highly publicized. Managers may wish to consider new product introductions stressing effective but simple formulations to this market segment, perhaps by using a well-known family brand name (if they possess one) since the second dimension appears to relate somewhat to awareness/market share. If the attributes/features of a new brand (such as those listed in Table 1) are known, predictions of where in this space the new brand would appear by means of the property fitting regression coefficients can be derived. In this way market share predictions can be obtained. In the next section, some other marketing extensions are discussed.

\section{DISCUSSION}

We have completed our discussion of the two multidimensional spatial choice models, including the presentation of an illustration with paired comparisons choice data collected over 14 brands of OTC analgesics. At this point, a word seems appropriate concerning data collection for the purpose of fitting these models. While in an experimental setting it may be feasible to collect complete paired comparisons data from each consumer, even on all pairs of a reasonably large set of alternatives (say 10 to 20 ), in field trials or telephone interview situations this probably is not feasible. However, there are many areas in marketing research that are conducive to laboratory studies in which such data are relatively easily collected from well-motivated subjects (who are paid or provided with other incentives) willing to spend 30 minutes to an hour (or even longer in some cases) responding to such paired comparisons preference items in either questionnaire form or via real-time interaction using computer terminals. On the other hand, if 
Figure 1: Plot of II vs. I.

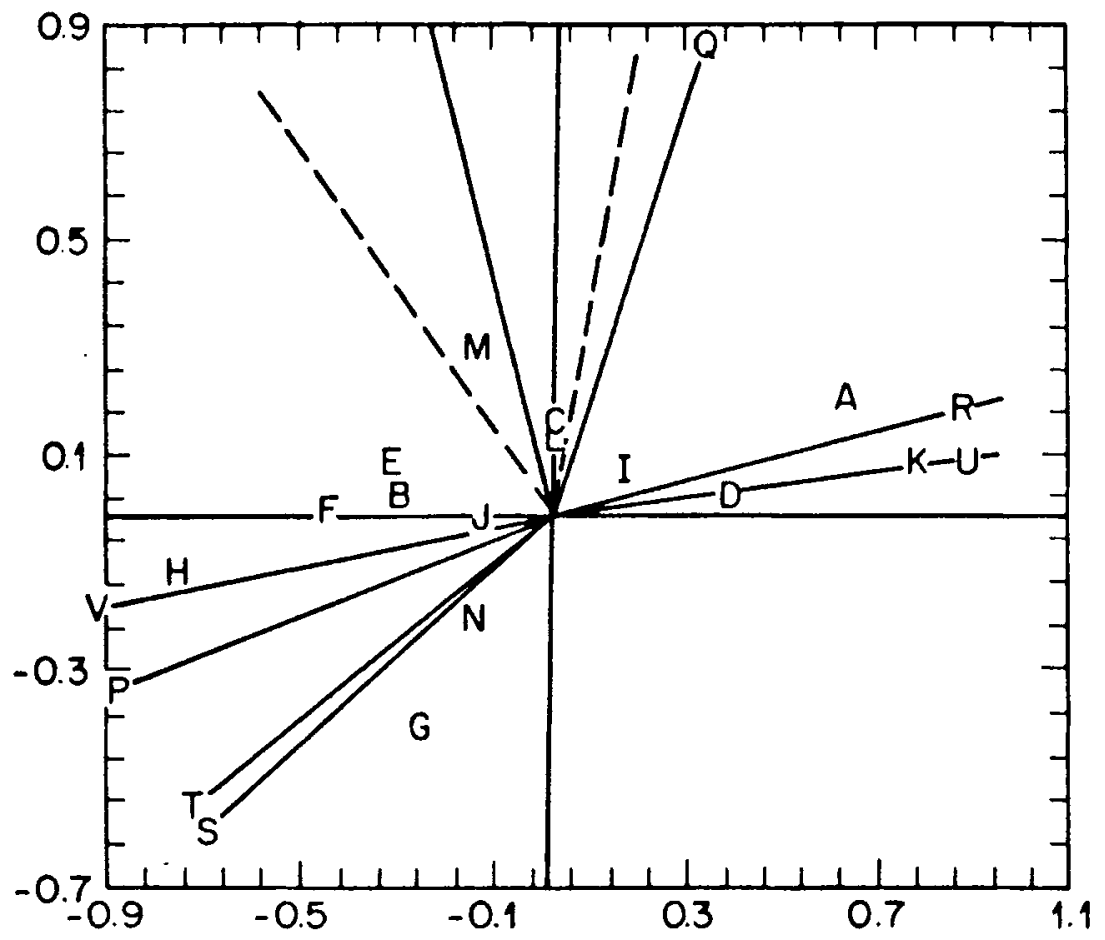

\begin{tabular}{lc}
\multicolumn{1}{c}{ Brands } & Label \\
\cline { 2 - 2 } Advil & A \\
Anacin & B \\
Anacin-3 & C \\
Ascriptin & D \\
Bayer & E \\
Bufferin & $\mathrm{F}$ \\
Cope & G \\
CVS (a generic) & $\mathrm{H}$ \\
Datril & $\mathrm{J}$ \\
Excedrin & $\mathrm{J}$ \\
Nuprin & $\mathrm{K}$ \\
Panadol & $\mathrm{L}$ \\
Tylenol & $\mathrm{M}$ \\
Vanquish & $\mathrm{N}$ \\
\hline
\end{tabular}

one is willing to treat different consumers as replications (as we have done in the analyses reported above on the "analgesics" data, even though complete paired comparisons data were collected from each student), it should be quite feasible to carry out analyses in terms of these models even with such relatively sparse data from each respondent. For many kinds of studies this assumption of interchangeability of subjects, implying a lack of systematic individual differences, appears quite reasonable (e.g., one faces homogeneous market segments). In such cases, the distributions of vector termini or ideal points are presumably composites of systematic, between-subject variation of centroids of vectors or of ideal points and within-subject variability of the type posited in the WV or WIP models, respectively. By using appropriately balanced, incomplete experimental designs, it is 
Table 3: Correlations between attributes and brand coordinates.

\begin{tabular}{lcc}
\hline & \multicolumn{2}{c}{ Dimensions } \\
\cline { 2 - 3 } Attributes & $\mathrm{I}$ & \multicolumn{1}{c}{$\mathrm{II}$} \\
\hline $\mathrm{P}$ & -.677 & -.703 \\
$\mathrm{Q}$ & .065 & .357 \\
$\mathrm{R}$ & .753 & .309 \\
$\mathrm{~S}$ & -.230 & -.513 \\
$\mathrm{~T}$ & -.263 & -.533 \\
$\mathrm{U}$ & .778 & .136 \\
$\mathrm{~V}$ & -.599 & -.362 \\
\hline
\end{tabular}

Legend for Attribute Labels

\begin{tabular}{lll}
\hline \multicolumn{1}{c}{ Attribute } & Label \\
\cline { 1 - 1 } $\mathrm{Mg}$ aspirin & $\mathrm{P}$ \\
$\mathrm{Mg}$ acetaminophen & $\mathrm{Q}$ \\
$\mathrm{Mg}$ ibuprofen & $\mathrm{R}$ \\
$\mathrm{Mg}$ caffeine & $\mathrm{S}$ \\
$\mathrm{Mg}$ buffered ingredients & $\mathrm{T}$ \\
Price & $\mathrm{U}$ \\
Maximum dosage per day & $\mathrm{V}$ \\
\hline
\end{tabular}

possible to collect complete paired comparisons from a sample of subjects (treated as replications).

When heterogeneous market segments are encountered, it should be possible to segment respondents on the basis of demand characteristics, demographic or psychographic information, and/or by use of some data-based clustering procedure. Subsets of respondents corresponding to these segments would then be defined, which could be treated as pseudosubjects in analyses to allow fitting of individual differences parameters (vector or ideal point centroids and/or covariance matrices) for these segmental pseudosubjects. When combined with appropriate computational algorithms for predicting probabilities of first choice from among larger sets of alternatives (ideally, separately within pseudosubject segments) and with appropriate use of the statistical tests and procedures for imposing constraints on the solution configurations, these models and associated methods should provide a very powerful methodology for both exploratory and confirmatory analyses of preferential choice data collected in marketing studies.

Another potentially valuable area of application concerns conjoint analysis studies where, in recently developed versions of the WV and WIP models, hypothetical brand coordinates can be specified as exact functions of the conjoint experimental design matrix [24]. Such explicit constraints might be useful in connection with designing new products (pricing plans) comprising different combinations of the feature sets. Such new product designs could be based on combinations of features (or feature levels) that correspond to theoretical stimulus positions predicted to be optimal from a marketing point of view on the basis of extrapolation from the fitted model(s) (i.e., to optimize market share, profitability, or some other well-defined objective) as performed in GENFOLD2 [27] [28]. [Received: August 4, 1988. Accepted: March 20, 1989.]

\section{REFERENCES}

[1] Akaike, H. On entropy maximization principle. In P. R. Krishnaiah (Ed.), Applications of statistics. Amsterdam: North-Holland, 1977. 
[2] Bechtel, G. G. Folded and unfolded scaling from preferential paired comparisons. Journal of Mathematical Psychology, 1968, 5, 333-357.

[3] Bechtel, G. G. Multidimensional preference scaling. The Hague: Mouton, 1976.

[4] Bechtel, G. G., Tucker, L. R., \& Chang, W. A scalar product model for the multidimensional scaling of choice. Psychometrika, 1971, 36, 369-388.

[5] Becker, G. M., DeGroot, M. H., \& Marschak, J. Probabilities of choice among very similar objects. Behavioral Science, 1963, 8, 306-311.

[6] Bennett, J. F., \& Hays, W. L. Multidimensional unfolding: Determining the dimensionality of ranked preference data. Psychometrika, 1964, 25, $27-43$.

[7] Bentler, P. M., \& Weeks, D. G. Restricted multidimensional scaling models. Journal of Mathematical Psychology, 1978, 17, 138-151.

[8] Bloxom, B. Constrained multidimensional scaling in N spaces. Psychometrika, 1978, 43, 397-408.

[9] Bock, R. D., \& Jones, L. V. The measurement and prediction of judgment and choice. San Franciscc, CA: Holden-Day, 1968.

[10] Box, B. E. P., Hunter, W. G., \& Hunter, J. S. Statistics for experiments. New York: Wiley, 1978.

[11] Bradley, R. A., \& Terry, M. E. Rank analysis of incomplete block designs I. The Method of paired comparisons. Biometrika, 1952, 39, 324-345.

[12] Carroll, J. D. Models and methods for multidimensional analysis of preferential choice (or other dominance) data. In E.D. Lantermann \& H. Feger (Eds.), Similarity and choice. Bern, Switzerland: Huber, 1980.

[13] Carroll, J. D., \& DeSarbo, W. S. Two-way spatial models for modeling individual differences in preference. In E.C. Hirschman \& M.B. Holbrook (Eds.), Advances in consumer research (Vol. 12). Prov, UT: Association for Consumer Research, Brigham Young Graduate School of Management, 1975.

[14] Carroll, J. D., Pruzansky, S., \& Kruskal, J. B. CANDELINC: A general approach to multidimensional analysis of many-way arrays with linear constraints on parameters. Psychometrika, $1979,45,3-24$.

[15] Carroll, J. D., \& Winsberg, S. Maximum likelihood procedures for metric and quasi-nonmetric fitting of an extended INDSCAL model assuming both common and specific dimension. Paper presented at the Multidimensional Data Analysis Workshop, Cambridge, England, June 1985.

[16] Chow, G. C. A comparison of information and posterior probability criteria for model selection. Journal of Econometrics, 1981, 16, 21-33.

[17] Coombs, C. H. Psychological scaling without a unit of measurement. Psychological Review, 1950, 57, $148-158$

[18] Coombs, C. H. A theory of data. New York: Wiley, 1964.

[19] Coombs, C. H., Greenberg, M., \& Zinnes, J. A double law of comparative judgment for the analysis of preferential choice and similarities data. Psychometrika, 1961, 26, 165-171.

[20] Cooper, L. G., \& Nakanishi, M. Two logit models for external analysis of preference. Psychometrika, 1983, 48, 607-620.

[21] Daganzo, C. Multinomial probit. New York: Academic Press, 1980.

[22] David, H. A. The method of paired comparisons. New York: Hafner, 1963.

[23] DeSarbo, W. S., \& Carroll, J. D. Three-way metric unfolding via alternating weighted least squares. Psychometrika, 1985, 50, 275-300.

[24] DeSarbo, W. S., Carroll, J. D., Lehmann, D. R., \& O'Shaughnessy, J. Three-way multivariate conjoint anaiysis. Marketing Science, 1982, 1, 323-350.

[25] DeSarbo, W. S., De Soete, G., \& Eliashberg, J. A new stochastic multidimensional unfolding model for the investigation of paired comparison consumer preference/choice data. Journal of Economic Psychology, 1987, 8, 357-384.

[26] DeSarbo, W. S., Oliver, R. L., \& De Soete, G. A probabilistic multidimensional scaling vector model. Applied Psychological Measurement, 1986, IO(1), 79-98.

[27] DeSarbo, W. S., \& Rao, V. R. GENFOLD2: A set of models and algorithms for the GENeral unFOLDing analysis of preference/dominance data. Journal of Classification, 1984, 1, 147-186.

[28] DeSarbo, W. S., \& Rao, V. R. A constrained unfolding model for product positioning. Marketing Science, 1986, 5, 1-19.

[29] De Soete, G. On the relation between two generalized cases of Thurstone's law of comparative judgment. Mathématiques et Sciences Humaines, 1983, 81, 47-57.

[30] De Soete, G., \& Carroll, J. D. A maximum likelihood method for fitting the wandering vector model. Psychometrika, 1983, 48, 553-566.

[31] De Soete, G., \& Carroll, J. D. Probabilistic multidimensional choice models for representing paired comparisons data. In E. Diday, Y. Escofier, L. Lebart, J. Pages, Y. Schektman, \& R. Tomassone (Eds.) Data analysis and informatics IV. Amsterdam: North-Holland, 1986. 
[32] De Soete, G., Carroll, J. D., \& DeSarbo, W. S. The wandering ideal point model: A probabilistic multidimensional unfolding model for paired comparisons data. Journal of Mathematical Psycho$\log y, 1986,30,28-41$.

[33] Edgell, S. E., \& Geisler, W. S. A set-theoretic, random utility model of choice behavior. Journal of Mathematical Psychology, 1980, 21, 265-278.

[34] Fechner, G. T. Elemente der psychophysik. Leipzig: Breitkopf and Hartel, 1860.

[35] Halff, H. M. Choice theories for differentially comparable alternatives. Journal of Mathematical Psychology, 1976, 14, 244-246.

[36] Heiser, W. J., \& de Leeuw, J. Multidimensional mapping of preference data. Mathématiques et Sciences Humaines, 1981, 73, 39-96.

[37] Krantz, D. H. Rational distance function for multidimensional scaling. Journal of Mathematical Psychology, 1967, 4, 226-245.

[38] Luce, R. D. Individual choice behavior: A theoretical analysis. New York: Wiley, 1959.

[39] Marley, A. A. Multivariate stochastic processes compatible with 'aspect' models of similarity and choice. Psychometrika, 1981, 46, 421-428.

[40] McFadden, D. Econometric models of probabilistic choice. In C. Manski \& D. McFadden (Eds.), Structural analysis of discrete data. Cambridge: Massachussetts Institute of Technology Press, 1981.

[41] McFadden, D. Econometric analysis of qualitative response models. In Z. Griliches and $\mathbf{M}$. Intriligator (Eds.), Handbook of econometrics (Vol. 2). Amsterdam: North-Holland, 1984.

[42] McFadden, D. The choice theory approach to market research. Marketing Science, 1986, 5, 275. 297.

[43] Restle, F. Psychology of judgment and choice: A theoretical essay. New York: Wiley, 1961.

[44] Rumelhart, D. L., \& Greeno, J. G. Similarity between stimuli: An experimental test of the Luce and Restle choice models. Journal of Mathematical Psychology, 1971, 8, 370-381.

[45] Schönemann, P. H., \& Wang, M. M. An individual difference model for the multidimensional analysis of preference data. Psychometrika, 1972, 37, 275-305.

[46] Schwarz, G. Estimating the dimensions of a model. Annals of Statistics, 1978, 6, 461-464.

[47] Sixt], F. Probabilistic unfolding. Psychometrika, 1973, 38, 235-248.

[48] Sjöberg, L. Uncertainty of comparative judgment and multidimensional structure. Multivariate Behavioral Research, 1975, 10, 207-218.

[49] Sjöberg, L. Choice frequency and similarity. Scandinavian Journal of Psychology, 1977, 18, 103 115.

[50] Sjöberg, L. Similarity and correlation. In E.D. Lantermann \& H. Feger (Eds.), Similarity and choice. Bern, Switzerland: Huber, 1980.

[51] Sjöberg, L., \& Cappoza, D. Preference and cognitive structure of italian political parties. Italian Journal of Psychology, 1975, 2, 391-402.

[52] Slater, P. The analysis of personal preferences. British Journal of Statistical Psychology, 1960, 13, 119-135.

[53] Strauss, D. Choice by features: An extension of Luce's choice model to account for similarities. British Journal of Mathematical and Statistical Psychology, 1981, 22, 188-196.

[54] Takane, Y. Maximum likelihood estimation in the generalized case of Thurstone's model of comparative judgment. Japanese Psychological Research, 1980, 22, 188-196.

[55] Thurstone, L. L. A law of comparative judgment. Psychological Review, 1927, 34, 273-286.

[56] Torgerson, W. S. Theory and methods of scaling. New York: Wiley, 1958.

[57] Tucker, L. R. Intra-individual and inter-individual multidimensionality. In H. Gulliksen \& S. Messick (Eds.), Psychological scaling: Theory and applications. New York: Wiley, 1960.

[58] Tversky, A. Elimination by aspects: A theory of choice. Psychological Review, 1972, 79, 281-299.

[59] Tversky, A., \& Russo, J. E. Substitutability and similarity in binary choices. Journal of Mathematical Psychology, 1969, 6, 1-12.

[60] Tversky, A., \& Sattath, S. Preference trees. Psychological Review, 1979, 86, 542-573.

[61] Wang, M. M., Schönemann, P. H., \& Rusk, J. G. A conjugate gradient algorithm for the multidimensional analysis of preference data. Multivariate Behavioral Research, 1975, 10, 45-99.

[62] Winsberg, S., \& Carroll, J. D. A metric and quasi-nonmetric method for fitting an extended Euclidean model postulating both common and specific dimensions. Psychometrika, in press.

[63] Zinnes, J. L., \& Griggs, R. A. Probabilistic, multidimensional unfolding analysis. Psychometrika, $1974,39,327 \cdot 350$.

J. Douglas Carroll is a member of the technical staff at AT\&T Bell Laboratories in the Information Principles Research Laboratory. He received his B.S. degree in mathematics from the University 
of Florida and his Ph.D. in psychology from Princeton University. Dr. Carroll has published widely in such journals as Psychometrika, Journal of Classification, Journal of Mathematical Psychology, Journal of Marketing Research, Marketing Science, and Journal of Marketing as well as in a number of books and other publications. His research interests lie primarily in theory, methods and applications of multidimensional scaling and related methodology for analysis of data arising in psychology, marketing, and other social and behavioral sciences. Dr. Carroll is a member of the Society of Mathematical Psychology, the Psychonomic Society, the Eastern Psychological Association, Sigma Xi, and Phi Beta Kappa.

Geert De Soete is a Research Associate at the University of Ghent, Belgium. He received his M.A. in quantitative psychology from the University of North Carolina at Chapel Hill and his Ph.D. in theoretical and experimental psychology from the University of Ghent, Belgium. Dr. De Soete's main research interests are data analysis and choice modeling. He has published in Psychometrika, Journal of Mathematical Psychology, Journal of Classification, Applied Psychological Measurement, Applied Stochastic Models and Data Analysis, and others. He also edited (with Feger and Klauer) Recent Developments in Psychological Choice Modeling.

Wayne S. DeSarbo is Professor of Marketing and Statistics at the Graduate School of Business of the University of Michigan. He received his B.S. degree in economics from the Wharton School of the University of Pennsylvania and his M.A. degrees in sociology, administrative science/O.R., and marketing from Yale University and the University of Pennsylvania. Dr. DeSarbo obtained his Ph.D. in marketing and statistics from the University of Pennsylvania. He has published over 70 articles in such journals as the Journal of Marketing Research, Psychometrika, Journal of Consumer Research, Journal of Mathematical Psychology, Marketing Science, and Decision Sciences. Dr. DeSarbo's methodological interests lie in multidimensional scaling, classification, and multivariate statistics, especially as they pertain to substantive marketing problems in positioning, market structure, consumer choice, market segmentation, and competitive strategy. He is a member of AMA, ORSA/TIMS, Psychometric Society, ASA RSS, Classification Society, INSNA, IMS, Econometric Society, ACR, Decision Sciences Institute, Society of Multivariate Experimental Psychology, and Division 23 of APA. 